\section{PWE-103 PLK-1 IS UPREGULATED IN BARRETT'S ADENOCARCINOMA AND IS A SURROGATE MARKER OF ANEUPLOIDY}

doi:10.1136/gut.2011.239301.366

J M Dunn, ${ }^{1, *}$ S A McDonald, ${ }^{2}$ D Oukrif, ${ }^{3}$ R J Haidry, ${ }^{1}$ M A Butt, ${ }^{1}$ E Lovat, ${ }^{1}$ L B Lovat, ${ }^{1}$ G H Williams, ${ }^{3}$ M R Novelli ${ }^{3}{ }^{1}$ National Medical Laser Centre, University College London, London, UK; ${ }^{2}$ Centre for Gastroenterology, Barts and the London School of Medicine and Dentistry, London, UK; ${ }^{3}$ Histopathology, University College London, London, UK

Introduction DNA ploidy abnormalities, measured by flow or image cytometry, are markers of genomic instability that inform on future cancer risk in patients with Barrett's oesophagus (BO). Barriers to routine clinical use include cost, availability and interlaboratory reproducibility. DNA replication licensing factors (RLFs) ensure precise duplication of the genome and contribute to genomic stability, and may therefore act as surrogate markers of DNA ploidy abnormalities. RLFs have great potential for routine clinical use as they are measured by immunohistochemistry (IHC).

Aims To establish a surrogate IHC marker for DNA ploidy that can be utilised as a high throughput screening tool for the assessment of cancer risk in $\mathrm{BO}$.

Methods Oesophagectomy specimens from 10 patients with Barrett's adenocarcinoma were evaluated. A total of 54 areas were marked representing the metaplasia-dysplasia-carcinoma sequence. These were removed by laser capture microdissection (LCM) and processed for image cytometry DNA ploidy analysis (ICDA). Serial $4 \mu \mathrm{m}$ sections were cut and stained for 5 RLFs by standard immunohistochemistry protocols, and the labelling index of percentage positive cells (LI) was calculated for each marked area. These scores were then correlated with histology and DNA ploidy abnormalities.

Results There was a significant difference in the LI of geminin, cdc7 kinase and Polo like kinase 1 (PLK-1) between nondysplastic $\mathrm{BO}$ and HGD/Cancer. There was no significant difference between these two groups for KI67 or mcm2. PLK-1 had the highest degree of correlation with DNA ploidy status with a Pearson coefficient $\mathrm{r}^{2}=0.776(\mathrm{p}<0.01)$. When using a cut off score of $30 \%$, the sensitivity and specificity of PLK-1 for the detection of aneuploidy was $86 \%$ and $100 \%$ respectively.

Conclusion This is the first time geminin, cdc7 kinase and PLK-1 have been shown to be upregulated in Barrett's dysplasia and adenocarcinoma. PLK-1, a key regulator of mitosis, correlated most closely with aneuploidy measured on LCM tissue and holds great potential as a biomarker in $\mathrm{BO}$.

Competing interests None.

Keywords aneuploidy, Barrett's adenocarcinoma, immunohistochemistry, replication licensing factor. 